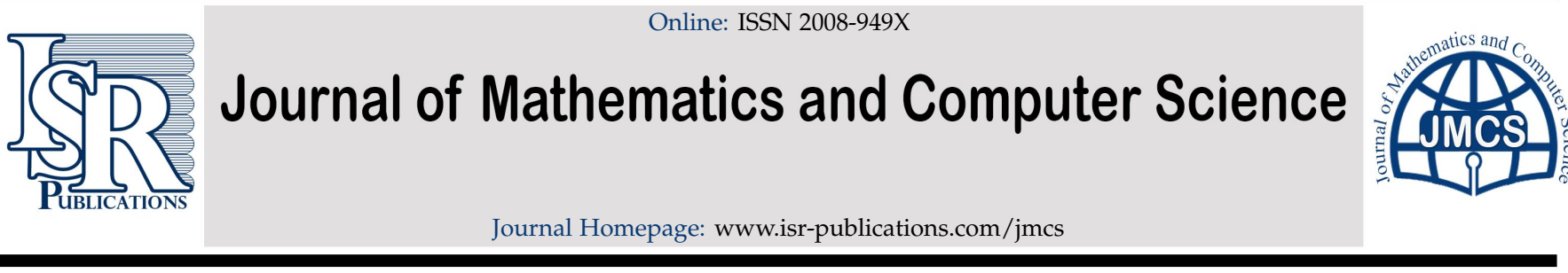

\title{
Semiconformal symmetry-A new symmetry of the space- time manifold of the general relativity
}

\author{
Naeem Ahmad Pundeer ${ }^{a, *}$, Musavvir Ali ${ }^{\mathrm{a}}$, Naeem Ahmad ${ }^{\mathrm{b}}$, Zafar Ahsan $^{\mathrm{c}}$ \\ ${ }^{a}$ Department of Mathematics, Aligarh Muslim University, India. \\ ${ }^{b}$ Department of Mathematics, Unaizah Engineering College, Unaizah, Qassim University, Al-Qassim, KSA. \\ ${ }^{c}$ Department of Mathematics, Maulana Azad National Urdu University, Hyderabad, India.
}

\begin{abstract}
In this paper, we have introduced a new symmetry property of space-time which is named as semiconformal curvature collineation, and its relationship with other known symmetry properties has been established. This new symmetry property of the space-time has also been studied for non-null and null electromagnetic fields.
\end{abstract}

Keywords: Curvature tensor, symmetries, electromagnetic fields.

2010 MSC: 53B20, 53B50, 83C20, 83C50.

(C)2020 All rights reserved.

\section{Introduction}

In recent years general relativists have been much interested in symmetries of space-time. Such interest is due to the need to simplify the Einstein's field equations in the search for their exact solutions. These geometrical symmetries of the space-time are often defined through the vanishing of the Lie derivative of certain tensors with respect to a vector (this vector may be time-like, space-like or null). The symmetries in general theory of relativity have been introduced by Katzin et al. in the papers $[14,15]$. These symmetries which are also known as collineations, were further studied by Ahsan [2-5], Ahsan and Ali [4-8] and Ali and Ahsan [9] among many others. However, in this paper our study is focused on these symmetries which can be used as simplifying assumptions in the exact solution of Einstein's field equations (EFE) but solving EFE by our findings will be the next target. Main objective of this paper is to give new symmetry in mathematical approach and analyse it on parameters of the well established literature on symmetries of space-time manifolds.

We know that two Riemannian spaces are conformally related through the equation $g_{\tilde{b}_{c}}=e^{2 \psi} g_{b c}$, where $g_{b c}$ and $g_{b c}$ are the two metric tensors of the two Riemannian spaces $\tilde{V}$ and $V$, while $\psi$ is a real

\footnotetext{
*Corresponding author

Email addresses: napundeer@myamu.ac.in (Naeem Ahmad Pundeer), musavvirali.maths@amu.ac.in (Musavvir Ali),

naeem.math@gmail.com (Naeem Ahmad), zafar.ahsan@rediffmail.com (Zafar Ahsan)
}

doi: $10.22436 /$ jmcs.020.03.07

Received: 2019-10-04 Revised: 2019-11-27 Accepted: 2019-12-12 
function of coordinates. Moreover, it is known that a harmonic function is defined as a function whose Laplacian vanishes. In general, a harmonic function is not transformed into a harmonic function. The conditions under which the harmonic functions remains invariant have been studied by Ishii [13]. He has introduced the conharmonic transformation as a subgroup of the conformal transformation satisfying the condition $\psi_{\cdot \mathrm{b}}^{\mathrm{b}}+\psi_{; \mathrm{b}} \psi_{;}^{\mathrm{b}}=0$. A rank four tensor $\mathrm{L}_{\mathrm{bcd}}^{\mathrm{h}}$ that remains invariant under conharmonic transformation for an $n$-dimensional Riemannian manifold $\left(M^{n}, g\right)$ of dimension $n \geqslant 4$, as follows:

$$
L_{b c d}^{h}=R_{b c d}^{h}+\frac{1}{n-2}\left(\delta_{c}^{h} R_{b d}-\delta_{d}^{h} R_{b c}+g_{b d} R_{c}^{h}-g_{b c} R_{d}^{h}\right),
$$

where $R_{b c d}^{h}, R_{b d}$ are Riemann curvature tensor and Ricci tensor respectively. The geometric properties of conharmonic curvature tensor have been discussed by Shaikh and Hui [22], while the relativistic significance of this tensor has been investigated by Abdussattar and Dwivedi [1] and Siddiqui and Ahsan [23]. In 2017, J. Kim [17] introduced curvature-like tensor field which remain invariant under conharmonic transformation. He named this new tensor as semiconformal curvature tensor and denoted it by $\mathrm{P}_{\mathrm{bcd}}^{\mathrm{h}}$. For a Riemannian manifold $M^{n}$ with metric $g$, this tensor is defined as (see also [18])

$$
P_{b c d}^{h}=-(n-2) B C_{b c d}^{h}+[A+(n-2) B] L_{b c d}^{h},
$$

provided the constants A and B are not simultaneously zero, is conformal curvature tensor defined as

$$
C_{b c d}^{h}=R_{b c d}^{h}+\frac{1}{n-2}\left(\delta_{c}^{h} R_{b d}-\delta_{d}^{h} R_{b c}+g_{b d} R_{c}^{h}-g_{b c} R_{d}^{h}\right)+\frac{R}{(n-1)(n-2)}\left(\delta_{d}^{h} g_{b c}-\delta_{c}^{h} g_{b d}\right),
$$

where $R_{a b}$ is the Ricci tensor and $R$ is the scalar curvature. For a special substitution $A=1$ and $B=\frac{-1}{(n-2)}$, the semiconformal curvature tensor reduces to conformal curvature tensor, while for $A=1$ and $B=0$, it reduces to conharmonic curvature tensor. It can be noted that throughout the paper we will be assuming that $A \neq 0$ and $B \neq 0$. The semiconformal curvature $P_{h b c d}$ satisfies the following symmetry properties

$$
P_{h b c d}=-P_{b h c d}=-P_{h b d c}=P_{c d h b}, \quad \text { and } \quad P_{h b c d}+P_{c h b d}+P_{b c h d}=0 .
$$

In this paper, we define a new symmetry in terms of semiconformal curvature tensor and study its relationship with other symmetries of the space-time. We call this new symmery as semiconformal curvature collineation. Section 2 contains some known results that are required for our investigation. In sections 2 and 4, the relationship between semiconformal curvature collineation and the other symmetry properties for a general Riemannian space and for a Riemannian space with vanishing Ricci tensor, respectively, have been established. Finally in section 5, the semiconformal curvature collineation has been studied for non-null and null electromagnetic fields.

\section{Preliminaries}

A geometrical symmetry of the space-time is often defined in terms of the Lie derivative of a tensor. These symmetries are also known as collineations. The literature on such collineations is very large and still expanding with results of elegance. Here we shall mention only those symmetry assumptions that are necessary for our study and we have (cf. [12, 21, 28])

Definition 2.1 (Motion (M)). A space-time is said to admit motion if there exists a vector field $\xi^{a}$ such that $^{1}$

$$
\eta_{\mathrm{ab}} \equiv \mathcal{L}_{\xi} \mathrm{g}_{\mathrm{ab}}=\xi_{\mathrm{a} ; \mathrm{b}}+\xi_{\mathrm{b} ; \mathrm{a}}=0 .
$$

\footnotetext{
${ }^{1}$ Indices take the values $1,2,3, \ldots, n$ and the summation convention is used. Covariant differentiation is indicated by a semicolon (;) and partial differentiation by a comma (,).
} 
Equation (2.1) is known as Killing equation and the vector $\xi^{\mathrm{a}}$ is known as a Killing vector field.

Definition 2.2 (Affine Collineation (AC)). A space-time is said to admit an affine collineation if there is a vector field $\xi^{\mathrm{a}}$ such that

$$
\mathcal{L}_{\xi} \Gamma_{\mathrm{ab}}^{\mathrm{c}} \equiv \frac{1}{2} g^{\mathrm{cd}}\left(\eta_{\mathrm{d} a ; b}+\eta_{\mathrm{db} ; \mathrm{a}}-\eta_{\mathrm{ab} ; \mathrm{d}}\right)=0,
$$

where $\Gamma_{a b}^{c}$ is the Christoffel symbol of second kind. Hence the necessary and sufficient condition for an $\mathrm{AC}$ [from equation (2.2)] is

$$
\eta_{\mathrm{ab} ; \mathrm{c}}=0,
$$

It may be noted, from equations (2.2) and (2.3), that every $\mathrm{M}$ is AC.

Definition 2.3 (Conformal Motion (Conf M)). A space-time is said to admit a conformal motion if there exists a vector field $\xi^{a}$ such that

$$
\eta_{a b}=2 \phi g_{a b}
$$

where $\phi$ is scalar and we may express it in the following form

$$
\phi=\frac{1}{4} \xi_{;}^{\mathrm{d}}
$$

Definition 2.4 (Projective Collineation (PC)). A space-time is said to admit projective collineation if there exists a vector $\xi^{\mathrm{a}}$ such that

$$
\mathcal{L}_{\xi} \prod_{\mathrm{bc}}^{\mathrm{a}}=0,
$$

where the projective connection [25] is defined as for $n=4$

$$
\prod_{b c}^{a}=\Gamma_{b c}^{a}-\frac{1}{5}\left(\delta_{b}^{a} \Gamma_{h c}^{h}+\delta_{c}^{a} \Gamma_{h b}^{h}\right) .
$$

From equations (2.5) and (2.6), we get

$$
\mathcal{L}_{\xi} \Gamma_{\mathrm{bc}}^{\mathrm{a}}=\delta_{\mathrm{b}}^{\mathrm{a}} \sigma_{; \mathrm{c}}+\delta_{\mathrm{c}}^{\mathrm{a}} \sigma_{; \mathrm{b}}
$$

where

$$
\sigma_{; \mathrm{c}}=\frac{1}{5} \xi_{; m \mathrm{~m}}^{\mathrm{m}}
$$

Further, for every projective collineation, we have [14]

$$
\mathcal{L}_{\xi} W_{\mathrm{bcd}}^{\mathrm{h}}=0,
$$

where the Weyl projective curvature tensor for $n=4$ is given by

$$
W_{b c d}^{h}=R_{b c d}^{h}-\frac{1}{3}\left(\delta_{d}^{h} R_{b c}-\delta_{c}^{h} R_{b d}\right) .
$$

From equations (2.7), (2.8), and (2.9) it follows that every AC is PC.

Definition 2.5 (Conformal Collineation (Conf C)). A space-time is said to admit a conformal collineation if there exists a vector $\xi^{a}$ such that

$$
\mathcal{L}_{\xi} \Gamma_{\mathrm{bc}}^{\mathrm{a}}=\delta_{\mathrm{b}}^{\mathrm{a}} \phi_{; \mathrm{c}}+\delta_{\mathrm{c}}^{\mathrm{a}} \phi_{; \mathrm{b}}-\mathrm{g}_{\mathrm{bc}} \mathrm{g}^{\mathrm{am}} \phi_{; \mathrm{m}}
$$

where $\phi=\frac{1}{4} \xi_{; \mathrm{d}}^{\mathrm{d}}$. Equations (2.4) and (2.11) may be expressed as [14]

$$
\eta_{\mathrm{ab} ; \mathrm{c}}=2 \phi_{; \mathrm{c}} \mathrm{g}_{\mathrm{ab}}
$$


and that every Conf C must satisfy (for explanation c.f., [28])

$$
\mathcal{L}_{\xi} C_{\mathrm{bcd}}^{\mathrm{h}}=0,
$$

where $C_{b c d}^{h}$ is conformal curvature tensor, which from equation (1.2) for $n=4$, is given by

$$
C_{b c d}^{h}=R_{b c d}^{h}+\frac{1}{2}\left(\delta_{c}^{h} R_{b d}-\delta_{d}^{h} R_{b c}+g_{b d} R_{c}^{h}-g_{b c} R_{d}^{h}\right)+\frac{R}{6}\left(\delta_{d}^{h} g_{b c}-\delta_{c}^{h} g_{b d}\right)
$$

Definition 2.6 (Curvature Collineation (CC)). A space-time is said to admit curvature collineation if there is a vector field $\xi^{a}$ such that

$$
\mathcal{L}_{\xi} R_{b c d}^{h}=0,
$$

where Riemann curvature tensor is defined as [12]

$$
\mathrm{R}_{\mathrm{bcd}}^{\mathrm{h}}=\Gamma_{\mathrm{hd}, \mathrm{c}}^{\mathrm{h}}-\Gamma_{\mathrm{hc}, \mathrm{d}}^{\mathrm{h}}+\Gamma_{\mathrm{bd}}^{\mathrm{m}} \Gamma_{\mathrm{mc}}^{\mathrm{h}}-\Gamma_{\mathrm{bc}}^{\mathrm{m}} \Gamma_{\mathrm{md}}^{\mathrm{h}} .
$$

Definition 2.7 (Ricci Collineation (RC)). A space-time is said to admit Ricci collineation if there is a vector field $\xi^{\mathrm{a}}$ such that

$$
\mathcal{L}_{\xi} R_{a b}=0
$$

where $R_{a b}$ is the Ricci tensor.

Definition 2.8 (Maxwell collineation (MC)). The electromagnetic field inherits the symmetry property of space-time such that

$$
\mathcal{L}_{\xi} \mathrm{F}_{\mathrm{ab}}=\mathrm{F}_{\mathrm{ab} ; \mathrm{c}} \xi^{\mathrm{c}}+\mathrm{F}_{\mathrm{ac}} \xi_{; \mathrm{b}}^{\mathrm{c}}+\mathrm{F}_{\mathrm{bc}} \xi_{; ; \mathrm{a}}^{\mathrm{c}}=0,
$$

where $F_{a b}$ is the electromagnetic field tensor. Such symmetry of space-time is called Maxwell collineation [10].

\section{Semiconformal symmetry}

For $n=4$, the semiconformal curvature tensor, from equation (1.1), is given by

$$
P_{b c d}^{h}=-2 B C_{b c d}^{h}+[A+2 B] L_{b c d}^{h},
$$

where $C_{b c d}^{h}$ and $L_{b c d}^{h}$ are the conformal and conharmonic curvature tensor, respectively. We now define a new symmetry for the space-time manifold of general relativity as

Definition 3.1 (Semiconformal Curvature Collineation (Semiconf CC)). A space-time $V_{4}$ is said to admit a semiconformal curvature collineation if there exists a vector field $\xi^{a}$ such that

$$
\mathcal{L}_{\xi} P_{b c d}^{h}=0,
$$

where $P_{b c d}^{h}$ is semiconformal curvature tensor is defined in equation (1.1).

We also define the following definition.

Definition 3.2 (Conharmonic Curvature Collineation (Conh CC)). A space-time $V_{4}$ is said to admit a conharmonic curvature collineation if there exists a vector $\xi^{a}$ such that

$$
\mathcal{L}_{\xi} \mathrm{L}_{\mathrm{bcd}}^{\mathrm{h}}=0,
$$

where conharmonic curvature tensor $\mathrm{L}_{\mathrm{bcd}}^{\mathrm{h}}$ is defined by [23]

$$
L_{b c d}^{h}=R_{b c d}^{h}+\frac{1}{2}\left(\delta_{c}^{h} R_{b d}-\delta_{d}^{h} R_{b c}+g_{b d} R_{c}^{h}-g_{b c} R_{d}^{h}\right) .
$$


Before giving the next definition we should have an idea of concircular transformation. In general conformal transformation $\left(g \tilde{b} c=\psi^{2} g_{b c}\right)$ does not transform the geodesic circles to the geodesic circles. Therefore in 1940, Yano [27] introduced a transformation which preserves the geodesic circles. Actually Yano used the conformal transformation together with the condition $\psi_{; \mathrm{b} ; \mathrm{c}}=\alpha g_{\mathrm{bc}}$ and named it as concircular transformation. He defines these symmetry property as following.

Definition 3.3 (Concircular Curvature Collineation (Conc CC)). A space-time $V_{4}$ is said to admit a concircular curvature collineation if there exists a vector $\xi^{a}$ such that $\mathcal{L}_{\xi} M_{\mathrm{bcd}}^{\mathrm{h}}=0$, where concircular curvature tensor $M_{b c d}^{h}$ is defined by [28]

$$
M_{b c d}^{h}=R_{b c d}^{h}-\frac{R}{12}\left(\delta_{d}^{h} g_{b c}-\delta_{c}^{h} g_{b d}\right) .
$$

Using equations (2.12) and (3.4) in equation (3.1), we get

$$
P_{b c d}^{h}=A\left[R_{b c d}^{h}+\frac{1}{2}\left(\delta_{c}^{h} R_{b d}-\delta_{d}^{h} R_{b c}+g_{b d} R_{c}^{h}-g_{b c} R_{d}^{h}\right)\right]-\frac{B R}{3}\left(\delta_{d}^{h} g_{b c}-\delta_{c}^{h} g_{b d}\right) .
$$

Theorem 3.4. The necessary and sufficient condition for a semiconformal curvature collineation (Semiconf CC) to be a curvature collineation (CC) is $\phi_{; \mathrm{bc}}=0$, where $\phi=\frac{1}{4} \xi_{; \mathrm{d}}^{\mathrm{d}}$ is a scalar function.

Proof. The semiconformal curvature tensor in terms of Riemann, Ricci and scalar curvature tensors is given in equation (3.6). For semiconformal curvature collineation to be reduced to curvature collineation, we first suppose that $V_{4}$ admits a semiconformal curvature collineation.

We know the Christoffel symbols vanish but not their derivative at a point in geodesic coordinate system. Therefore, at this point equation (2.13) leads to

$$
\mathcal{L}_{\xi} \mathrm{R}_{\mathrm{bcd}}^{\mathrm{h}}=\left(\mathcal{L}_{\xi} \Gamma_{\mathrm{db}}^{\mathrm{h}}\right)_{; \mathrm{c}}-\left(\mathcal{L}_{\xi} \Gamma_{\mathrm{cb}}^{\mathrm{h}}\right)_{; \mathrm{d}}
$$

using equation (2.11), we have

$$
\begin{aligned}
& \mathcal{L}_{\xi} R_{b c d}^{h}=\left(\delta_{d}^{h} \phi_{; b}+\delta_{b}^{h} \phi_{; d}-g_{d b} g^{h m} \phi_{; m}\right)_{; c}-\left(\delta_{c}^{h} \phi_{; b}+\delta_{b}^{h} \phi_{; c}-g_{c b} g^{h n} \phi_{; n}\right)_{; d} \text { or } \\
& \mathcal{L}_{\xi} R_{b c d}^{h}=\left(\delta_{d}^{h} \phi_{; b c}+\delta_{b}^{h} \phi_{; d c}-g_{d b} g^{h m} \phi_{; m c}\right)-\left(\delta_{c}^{h} \phi_{; b d}+\delta_{b}^{h} \phi_{; c d}-g_{c b} g^{h n} \phi_{; n d}\right),
\end{aligned}
$$

to find the required condition for our target we have to put $\mathcal{L}_{\xi} R_{b c d}^{h}=0$, therefore equation (3.8) leads to

$$
\delta_{\mathrm{d}}^{\mathrm{h}} \phi_{; \mathrm{bc}}+\delta_{\mathrm{b}}^{\mathrm{h}} \phi_{; \mathrm{dc}}-g_{\mathrm{db}} g^{\mathrm{hm}} \phi_{; \mathrm{mc}}-\delta_{\mathrm{c}}^{\mathrm{h}} \phi_{; \mathrm{bd}}-\delta_{\mathrm{b}}^{\mathrm{h}} \phi_{; \mathrm{cd}}+g_{\mathrm{cb}} g^{\mathrm{hn}} \phi_{; \mathrm{nd}}=0,
$$

performing some indexing arrangement in the equation (3.9), we get

$$
2 \phi_{; b c}+g_{c b} g^{d n} \phi_{; n d}=0 .
$$

In equation (3.10), multiplied by $g^{b c}$, we get $g^{b c} \phi_{; b c}=0$. It implies that $\phi_{; b c}=0$, where $\phi=\frac{1}{4} \xi_{; d}^{d}$ is a scalar function. This completes the proof.

Theorem 3.5. The necessary and sufficient condition for a semiconformal curvature collineation to be a Weyl projective curvature collineation is that $\sigma_{; \mathrm{bc}}=0$, where $\sigma=\frac{1}{5} \mathrm{\xi} ; \mathrm{m}$.

Proof. From equations (2.10) and (3.6), the semiconformal curvature tensor in terms of Weyl projective curvature tensor may be expressed in the following way

$$
\begin{aligned}
& P_{b c d}^{h}=A\left[W_{b c d}^{h}+\frac{1}{6}\left(\delta_{c}^{h} R_{b d}-\delta_{d}^{h} R_{b c}\right)+\frac{1}{2}\left(g_{b d} R_{c}^{h}-g_{b c} R_{d}^{h}\right)\right]-\frac{B R}{3}\left(\delta_{d}^{h} g_{b c}-\delta_{c}^{h} g_{b d}\right), \text { or } \\
& P_{b c d}^{h}=A\left[W_{b c d}^{h}+\frac{1}{6}\left(\delta_{c}^{h} R_{b d j}^{j}-\delta_{d}^{h} R_{b c i}^{i}\right)+\frac{1}{2}\left(\delta_{d}^{h} R_{b c u}^{u}-\delta_{c}^{h} R_{b d t}^{t}\right)\right]-\frac{4 B}{3}\left(\delta_{d}^{h} R_{b c v}^{v}-\delta_{c}^{h} R_{b d w}^{w}\right) .
\end{aligned}
$$


For semiconformal curvature collineation to be reduced to Weyl projective curvature collineation, we start with a semiconformal curvature collineation admitted by $\mathrm{V}_{4}$, i.e., $\mathcal{L}_{\xi} \mathrm{P}_{\mathrm{bcd}}^{\mathrm{h}}=0$, equation (3.11) leads to

$$
\begin{aligned}
A \mathcal{L}_{\xi} W_{\mathrm{bcd}}^{\mathrm{h}}= & \frac{A}{6} \delta_{\mathrm{d}}^{\mathrm{h}} \mathcal{L}_{\xi} R_{\mathrm{bci}}^{\mathrm{i}}-\frac{\mathrm{A}}{6} \delta_{\mathrm{c}}^{\mathrm{h}} \mathcal{L}_{\xi} R_{\mathrm{bdj}}^{j}+\frac{A}{2} \delta_{\mathrm{c}}^{\mathrm{h}} \mathcal{L}_{\xi} R_{\mathrm{bdt}}^{\mathrm{t}}-\frac{\mathrm{A}}{2} \delta_{\mathrm{d}}^{\mathrm{h}} \mathcal{L}_{\xi} R_{\mathrm{bcu}}^{\mathrm{u}} \\
& +\frac{4 \mathrm{~B}}{3} \delta_{\mathrm{d}}^{\mathrm{h}} \mathcal{L}_{\xi} R_{\mathrm{bc} v}^{v}-\frac{4 \mathrm{~B}}{3} \delta_{\mathrm{c}}^{\mathrm{h}} \mathcal{L}_{\xi} \mathrm{R}_{\mathrm{bd} w}^{w}
\end{aligned}
$$

using the equations (3.7) and (3.12), we get

$$
\begin{aligned}
A \mathcal{L}_{\xi} W_{\mathrm{bcd}}^{\mathrm{h}}= & \frac{\mathrm{A}}{6} \delta_{\mathrm{d}}^{\mathrm{h}}\left[\left(\mathcal{L}_{\xi} \Gamma_{\mathrm{ib}}^{\mathrm{i}}\right)_{; \mathrm{c}}-\left(\mathcal{L}_{\xi} \Gamma_{\mathrm{cb}}^{\mathrm{i}}\right)_{; \mathrm{i}}\right]-\frac{\mathrm{A}}{6} \delta_{\mathrm{c}}^{\mathrm{h}}\left[\left(\mathcal{L}_{\xi} \Gamma_{j \mathrm{~b}}^{\mathrm{j}}\right)_{; \mathrm{d}}-\left(\mathcal{L}_{\xi} \Gamma_{\mathrm{db}}^{\mathrm{j}}\right)_{; j}\right] \\
& +\frac{\mathrm{A}}{2} \delta_{\mathrm{c}}^{\mathrm{h}}\left[\left(\mathcal{L}_{\xi} \Gamma_{\mathrm{tb}}^{\mathrm{t}}\right)_{; \mathrm{d}}-\left(\mathcal{L}_{\xi} \Gamma_{\mathrm{db}}^{\mathrm{t}}\right)_{; \mathrm{t}}\right]-\frac{\mathrm{A}}{2} \delta_{\mathrm{d}}^{\mathrm{h}}\left[\left(\mathcal{L}_{\xi} \Gamma_{\mathrm{ub}}^{\mathrm{u}}\right)_{; \mathrm{c}}-\left(\mathcal{L}_{\xi} \Gamma_{\mathrm{cb}}^{\mathrm{u}}\right)_{; \mathrm{u}}\right] \\
& +\frac{4 \mathrm{~B}}{3} \delta_{\mathrm{d}}^{\mathrm{h}}\left[\left(\mathcal{L}_{\xi} \Gamma_{v \mathrm{~b}}^{\mathrm{v}}\right)_{; \mathrm{c}}-\left(\mathcal{L}_{\xi} \Gamma_{\mathrm{cb}}^{v}\right)_{; v}\right]-\frac{4 \mathrm{~B}}{3} \delta_{\mathrm{c}}^{\mathrm{h}}\left[\left(\mathcal{L}_{\xi} \Gamma_{w \mathrm{~b}}^{\mathrm{w}}\right)_{; \mathrm{d}}-\left(\mathcal{L}_{\xi} \Gamma_{\mathrm{db}}^{\mathrm{w}}\right)_{; w}\right],
\end{aligned}
$$

now with the help of equation (2.7), the above equation will reduced to the following form

$$
A \mathcal{L}_{\xi} W_{b c d}^{h}=\frac{A}{2} \delta_{d}^{h} \sigma_{; b c}-\frac{A}{2} \delta_{c}^{h} \sigma_{; b d}+\frac{3 A}{2} \delta_{c}^{h} \sigma_{; b d}-\frac{3 A}{2} \delta_{d}^{h} \sigma_{; b c}+4 B \delta_{d}^{h} \sigma_{; b c}-4 B \delta_{c}^{h} \sigma_{; b d}
$$

Further for Weyl projective curvature collineation $\mathcal{L}_{\xi} W_{\mathrm{bcd}}^{\mathrm{h}}=0$ putting in (3.13) and contraction over $h$ and $d$, equation (3.13) leads to $(12 B-3 A) \sigma_{; b c}=0$, which implies that $\sigma_{; b c}=0$, where $\sigma=\frac{1}{5} \xi ; m$. This establishes the proof.

From Katzin et al. [14], we have the following.

Lemma 3.6. Every motion in $\mathrm{V}_{4}$ is curvature collineation (CC), Weyl projective collineation (WPC) and Weyl conformal collineation (W Conf $C$ ).

Also we can write the following.

Lemma 3.7. Every motion in $a \mathrm{~V}_{4}$ is a Conh CC and Conc CC.

Proof. Proof is obvious from Lemma 3.6 on use of the equations (3.3)-(3.5).

Theorem 3.8. A space-time $\mathrm{V}_{4}$ admits semiconformal curvature collineation along a vector field $\xi^{\mathrm{a}}$ provided that $\xi^{\mathrm{a}}$ is Killing.

Proof. From equations (3.4) and (3.6), the relation between semiconformal and conharmonic curvature tensor is given by

$$
P_{b c d}^{h}=A L_{b c d}^{h}-\frac{B R}{3}\left(\delta_{d}^{h} g_{b c}-\delta_{c}^{h} g_{b d}\right),
$$

taking the Lie derivative on both sides of (3.14), we have

$$
\begin{aligned}
\mathcal{L}_{\xi} P_{b c d}^{h}= & A \mathcal{L}_{\xi} L_{b c d}^{h}-\frac{B}{3}\left[\delta_{d}^{h} R \mathcal{L}_{\xi} g_{b c}+\delta_{d}^{h} g_{b c} \mathcal{L}_{\xi} R-\delta_{c}^{h} R \mathcal{L}_{\xi} g_{b d}-\delta_{c}^{h} g_{b d} \mathcal{L}_{\xi} R\right] \\
= & A \mathcal{L}_{\xi} L_{b c d}^{h}-\frac{B}{3}\left[\left(\xi^{m} R_{h ; m}^{h}-\xi_{; m}^{h} R_{h}^{m}+\xi_{; h}^{m} R_{m}^{h}\right) \delta_{d}^{h} g_{b c}+\left(\xi_{b ; c}+\xi_{c ; b}\right) \delta_{d}^{h} R\right. \\
& \left.-\left(\xi^{m} R_{h ; m}^{h}-\xi_{; m}^{h} R_{h}^{m}+\xi_{; h}^{m} R_{m}^{h}\right) \delta_{c}^{h} g_{b d}-\left(\xi_{b ; d}+\xi_{d ; b}\right) \delta_{c}^{h} R\right],
\end{aligned}
$$

from equations (2.1), Lemmas 3.6 and 3.7, the terms on right side of equation (3.15) vanishes, hence the proof is done. 
Moreover, equations (3.5) and (3.6) lead to the following relation between semiconformal and concircular curvature tensors

$$
P_{b c d}^{h}=A\left[M_{b c d}^{h}+\frac{R}{12}\left(\delta_{d}^{h} g_{b c}-\delta_{c}^{h} g_{b d}\right)+\frac{1}{2}\left(\delta_{c}^{h} R_{b d}-\delta_{d}^{h} R_{b c}+g_{b d} R_{c}^{h}-g_{b c} R_{d}^{h}\right)\right]-\frac{B R}{3}\left(\delta_{d}^{h} g_{b c}-\delta_{c}^{h} g_{b d}\right),
$$

which on taking the Lie derivative on both sides yields

$$
\begin{aligned}
\mathcal{L}_{\xi} P_{b c d}^{h}= & A\left[\mathcal{L}_{\xi} M_{b c d}^{h}+\frac{1}{12}\left(\xi^{m} R_{h ; m}^{h}-\xi_{; m}^{h} R_{h}^{m}+\xi_{; h}^{m} R_{m}^{h}\right) \delta_{d}^{h} g_{b c}+\left(\xi_{b ; c}+\xi_{c ; b}\right) \delta_{d}^{h} R\right. \\
& -\left(\xi^{m} R_{h ; m}^{h}-\xi_{; m}^{h} R_{h}^{m}+\xi_{; h}^{m} R_{m}^{h}\right) \delta_{c}^{h} g_{b d}-\left(\xi_{b ; d}+\xi_{d ; b}\right) \delta_{c}^{h} R-\frac{1}{2}\left(\xi_{b ; c}+\xi_{c ; b}\right) R_{d}^{h} \\
& -\frac{1}{2}\left(\xi^{m} R_{d ; m}^{h}-\xi_{; m}^{h} R_{d}^{m}+\xi_{;}^{m} R_{m}^{h}\right) g_{b c}+\frac{1}{2} \delta_{c}^{h}\left(\xi^{m} R_{b d ; m}+\xi^{m} R_{m d ; b}+\xi^{m} R_{b m ; d}\right) \\
& -\frac{1}{2} \delta_{d}^{h}\left(\xi^{m} R_{b c ; m}+\xi^{m} R_{m c ; b}+\xi^{m} R_{b m ; c}\right)+\frac{1}{2}\left(\xi_{b ; d}+\xi_{d ; b}\right) R_{c}^{h}+\frac{1}{2} g_{b d}\left(\xi^{m} R_{c ; m}^{h}\right. \\
& \left.\left.-\xi_{; m}^{h} R_{c}^{m}+\xi_{;}^{m} R_{m}^{h}\right)\right]-\frac{B}{3}\left[\left(\xi^{m} R_{h ; m}^{h}-\xi_{; m}^{h} R_{h}^{m}+\xi_{; h}^{m} R_{m}^{h}\right) \delta_{d}^{h} g_{b c}+\left(\xi_{b ; c}+\xi_{c ; b}\right) \delta_{d}^{h} R\right. \\
& \left.-\left(\xi^{m} R_{h ; m}^{h}-\xi_{; m}^{h} R_{h}^{m}+\xi_{; h}^{m} R_{m}^{h}\right) \delta_{c}^{h} g_{b d}-\left(\xi_{b ; d}+\xi_{d ; b}\right) \delta_{c}^{h} R\right] .
\end{aligned}
$$

Remark 3.9. We find that the Lemmas 3.6 and 3.7 together with equations (2.1) and (3.16) give the similar result as in Theorem 3.8.

\section{Semiconformal symmetry in empty space-time}

The Einstein field equations are given by

$$
R_{b c}-\frac{1}{2} g_{b c} R=-k T_{b c}
$$

where $R_{b c}$ is the Ricci tensor, $g_{b c}$ is the metric tensor, $T_{b c}$ is the energy momentum tensor, $R$ is the scalar curvature tensor and $k$ is the constant. Multiplying by $g^{b c}$ and using $g^{b c} g_{b c}=4$, equation (3.16) takes the form

$$
\mathrm{R}=\mathrm{kT} \text {. }
$$

from the equations (4.1) and (4.2), we get

$$
R_{b c}=k\left(T_{b c}-\frac{1}{2} g_{b c} T\right) .
$$

If $T_{b c}=0$, then $T=g^{b c} T_{b c}=0$, equation (4.3) yields

$$
\mathrm{R}_{\mathrm{bc}}=0,
$$

these equations are the field equations for empty space-time.

Theorem 4.1. In an Empty space-time $\mathrm{V}_{4}^{0}$ Lie derivative of semiconformal curvature tensor is identical to Lie derivative of Riemann curvature tensor for $\mathrm{A}=1$.

Proof. From equation (3.6), we have

$$
\begin{aligned}
P_{b c d}^{h}= & A\left[R_{b c d}^{h}+\frac{1}{2}\left(\delta_{c}^{h} R_{b d}-\delta_{d}^{h} R_{b c}+g_{b d} g^{h i} R_{i c}-g_{b c} g^{h j} R_{j d}\right)\right] \\
& -\frac{B g^{p q} R_{p q}}{3}\left(\delta_{d}^{h} g_{b c}-\delta_{c}^{h} g_{b d}\right)=A R_{b c d}^{h}-B \delta_{d}^{h} R_{b c} .
\end{aligned}
$$

Now using the equation (4.4), equation (4.5) leads to $P_{b c d}^{h}=A R_{b c d}^{h}$. The Lie derivative on both sides of this equation give the immediate proof of the theorem. 
Theorem 4.2. In empty space-time $\mathrm{V}_{4}^{0}$ the Lie derivatives of semiconformal curvature and Weyl projective curvature tensors are identical for $\mathrm{A}=1$.

Proof. From (3.11), we have

$$
\begin{aligned}
P_{b c d}^{h} & =A\left[W_{b c d}^{h}+\frac{1}{6}\left(\delta_{c}^{h} R_{b d}-\delta_{d}^{h} R_{b c}\right)+\frac{1}{2}\left(g_{b d} g^{h i} R_{i c}-g_{b c} g^{h j} R_{j d}\right)\right]-\frac{B g^{p q} R_{p q}}{3}\left(\delta_{d}^{h} g_{b c}-\delta_{c}^{h} g_{b d}\right) \\
& =A\left[W_{b c d}^{h}+\frac{1}{3}\left(\delta_{d}^{h} R_{b c}-\delta_{c}^{h} R_{b d}\right)\right]-4 B \delta_{d}^{h} R_{b c},
\end{aligned}
$$

using the result for empty space-time in equation (4.6) and then performing Lie derivative, we get

$$
\mathcal{L}_{\xi} \mathrm{P}_{\mathrm{bcd}}^{\mathrm{h}}=A \mathcal{L}_{\xi} W_{\mathrm{bcd}}^{\mathrm{h}} .
$$

This establishes the theorem.

From equation (4.7), we can state the following.

Corollary 4.3. A space-time $\mathrm{V}_{4}^{0}$ admits semiconformal curvature collineation if and only if it admits Weyl projective curvature collineation.

Remark 4.4. Making use of the equations (3.4), (3.5), (3.6), and (4.4) also following similar steps as in Theorems 4.1, 4.2, and Corollary 4.3, we find the similar kind of results for conharmonic and concircular curvature tensors.

\section{Semiconformal curvature collineation and electromagnetic fields}

It is known that in general relativity, the electromagnetism can be described through Maxwell's equation

$$
\mathrm{F}_{[\mathrm{ab} ; \mathrm{c}]}=0, \quad \mathrm{~F}_{; \mathrm{b}}^{\mathrm{ab}}=\mathrm{J}^{\mathrm{a}},
$$

where the skew-symmetric tensor $F_{a b}$ represents the electromagnetic field tensor and $J^{a}$ the current density. Moreover, we have defined the Einstein field equations in equation (4.1) and in presence of matter in equation (4.3). The energy-momentum tensor for an electromagnetic field is given by

$$
\mathrm{T}_{\mathrm{ab}}=-\mathrm{F}_{\mathrm{ac}} \mathrm{F}_{\mathrm{b}}^{\mathrm{c}}+\frac{1}{4} \mathrm{~g}_{\mathrm{ab}} \mathrm{F}_{\mathrm{pq}} \mathrm{F}^{\mathrm{pq}}
$$

which is symmetric tensor. Equation (5.1) leads to $T_{a}^{a}=T=0$ and thus the Einstein equation for a purely electromagnetic distribution is given by

$$
\mathrm{R}_{\mathrm{ab}}=k \mathrm{~T}_{\mathrm{ab}} \text {. }
$$

The geometrical symmetry defined by equation (2.14) along with the symmetry given by equation (2.1) has been the subject of interest for quite some time. Thus for example, for non-null electromagnetic fields Woolley [26] has shown that if equation (2.1) holds then $F_{a b}$ satisfies $\mathcal{L}_{\xi} F_{a b}=k(\alpha) F_{a b}$ for some constant $\mathrm{k}(\alpha), \alpha=1,2, \ldots, \mathrm{r}$; while Michalski and Wainwright [19] have shown that $\mathcal{L}_{\xi} \mathrm{g}_{\mathrm{ab}}=0$ implies $\mathcal{L}_{\xi} \mathrm{F}_{\mathrm{ab}}=0$ for non-null fields. On the other hand, for non-null fields, Duggal [11] has proved the converse part under certain conditions. Maxwell collineations have also been studied by Ahsan [2,3]. It is seen that for null electromagnetic fields neither MC is a consequence of Motion nor Motion is a consequence of Maxwell collineation. Moreover, using Newman-Penrose formalism, Ahsan [3] has obtained the conditions under which a null electromagnetic field may admit Maxwell collineation and Motion. The concept of Maxwell collineation was further extended as Maxwell Inheritance (MI) by Ahsan and Ahsan [5], who applied this concept to (i) the space-time solution corresponding to strong gravitational waves propagating in generalized electromagnetic universes and (ii) the algebraically general twist-free solution of EinsteinMaxwell equation for non-radiative electromagnetic fields. 
In 1986 Khlebnikov [16] has obtained the solutions for Einstein-Maxwell equations corresponding to the strong gravitational waves in the generalized electromagnetic universe. He used the technique of N.P. formalism [20] to obtain the solution in non-radiative electromagnetic fields. In his solution he took the first real null tetrad vector $l^{a}$ corresponding to geodesic and shear-free and the tetrad as parallelly propagated along $l^{a}$ also proved that the solution does not admit the Maxwell collineation. As another example we can refer the twist-free algebraically general solution given by Tariq and Tupper [24]. For this solution of Einstein-Maxwell equations in non-null electromagnetic fields together with the condition of coupling theorem Tariq and Tupper proved that their solutions also do not admit the Maxwell collineation. Motivated by above discussions, in this section, we shall investigate the role of semiconformal curvature collineation to the non-null and null electromagnetic fields.

Lemma 5.1 ([19]). In a non-null electromagnetic field, the Lie derivative of electromagnetic field tensor $\mathrm{F}_{\mathrm{ab}}$ with respect to a vector field $\xi$ vanishes, if $\xi$ is Killing vector.

Theorem 5.2. A non-null electromagnetic field admits semiconformal curvature collineation along a Killing vector field.

Proof. From the equations (5.1), (5.2), and (3.6), we have

$$
\begin{aligned}
P_{b c d}^{h}= & A R_{b c d}^{h}-\frac{k A}{2}\left(g_{b c} F_{m}^{h} F_{d}^{m}-\delta_{c}^{h} F_{b m} F_{d}^{m}\right)+\left(\delta_{d}^{h} F_{b m} F_{c}^{m}-g_{b d} F_{m}^{h} F_{c}^{m}\right) \\
& +\left(\frac{k A}{4} F_{i j} F^{i j}-\frac{B R}{3}\right)\left(\delta_{d}^{h} g_{b c}-\delta_{c}^{h} g_{b d}\right) .
\end{aligned}
$$

Taking the Lie derivative on both side of the equation (5.3), we get

$$
\begin{aligned}
& \mathcal{L}_{\xi} P_{b c d}^{h}=A \mathcal{L}_{\xi} R_{b c d}^{h}-\frac{k A}{2}\left[\mathcal{L}_{\xi}\left(g_{b c}\right) F_{m}^{h} F_{d}^{m}+g_{b c} \mathcal{L}_{\xi}\left(F_{m}^{h}\right) F_{d}^{m}+g_{b c} F_{m}^{h} \mathcal{L}_{\xi}\left(F_{d}^{m}\right)\right. \\
& \left.-\delta_{\mathrm{c}}^{h} \mathcal{L}_{\xi}\left(\mathrm{F}_{\mathrm{bm}}\right) \mathrm{F}_{\mathrm{d}}^{\mathrm{m}}-\delta_{\mathrm{c}}^{\mathrm{h}} \mathrm{F}_{\mathrm{bm}} \mathcal{L}_{\xi}\left(\mathrm{F}_{\mathrm{d}}^{\mathrm{m}}\right)\right]+\left[\delta_{\mathrm{d}}^{\mathrm{h}} \mathcal{L}_{\xi}\left(\mathrm{F}_{\mathrm{bm}}\right) \mathrm{F}_{\mathrm{c}}^{\mathrm{m}}+\delta_{\mathrm{d}}^{\mathrm{h}} \mathrm{F}_{\mathrm{bm}} \mathcal{L}_{\xi}\left(\mathrm{F}_{\mathrm{c}}^{\mathrm{m}}\right)\right. \\
& \left.-\mathcal{L}_{\xi}\left(g_{b d}\right) F_{m}^{h} F_{c}^{m}-g_{b d} \mathcal{L}_{\xi}\left(F_{m}^{h}\right) F_{c}^{m}-g_{b d} F_{m}^{h} \mathcal{L}_{\xi}\left(F_{c}^{m}\right)\right]+\left[\frac{k A}{4} \mathcal{L}_{\xi}\left(F_{i j}\right) F^{i j}\right. \\
& \left.+\frac{k A}{4} F_{i j} \mathcal{L}_{\xi}\left(F^{i j}\right)-\frac{B}{3} \mathcal{L}_{\xi}(R)\right]\left[\delta_{d}^{h} g_{b c}-\delta_{c}^{h} g_{b d}\right] \\
& +\left(\frac{k A}{4} F_{i j} F^{i j}-\frac{B R}{3}\right)\left[\delta_{d}^{h} \mathcal{L}_{\xi}\left(g_{b c}\right)-\delta_{c}^{h} \mathcal{L}_{\xi}\left(g_{b d}\right)\right] .
\end{aligned}
$$

Further, use of symmetry properties of metric tensor and electromagnetic field tensor (i.e., equations (2.1) and (2.14)) to equation (5.4) yields

$$
\begin{aligned}
\mathcal{L}_{\xi} P_{b c d}^{h}= & A \mathcal{L}_{\xi} R_{b c d}^{h}-\frac{k A}{2}\left[\left(\xi_{b ; c}+\xi_{c ; b}\right) F_{m}^{h} F_{d}^{m}+g_{b c}\left(F_{m ; \mu}^{h} \xi^{\mu}+F_{\mu}^{h} \xi_{; m}^{\mu}-F_{m}^{\mu} \xi_{; \mu}^{h}\right) F_{d}^{m}\right. \\
& +g_{b c} F_{m}^{h}\left(F_{d ; \mu}^{m} \xi^{\mu}+F_{\mu}^{m} \xi_{; d}^{\mu}-F_{d}^{\mu} \xi_{; \mu}^{m}\right)-\delta_{c}^{h}\left(F_{b m ; \mu} \xi^{\mu}+F_{b \mu} \xi_{; m}^{\mu}+F_{m \mu} \xi_{; j}^{\mu}\right) F_{d}^{m} \\
& \left.-\delta_{c}^{h} F_{b m}\left(F_{d ; \mu}^{m} \xi^{\mu}+F_{\mu}^{m} \xi_{; d}^{\mu}-F_{d}^{\mu} \xi_{; \mu}^{m}\right)\right]+\left[\delta_{d}^{h}\left(F_{b m ; \mu} \xi^{\mu}+F_{b \mu} \xi_{; m}^{\mu}+F_{m \mu} \xi_{; b}^{\mu}\right) F_{c}^{m}\right. \\
& +\delta_{d}^{h} F_{b m}\left(F_{c ; \mu}^{m}+F_{\mu}^{m} \xi_{;}^{\mu}-F_{c}^{\mu} \xi_{; \mu}^{m}\right)-\left(\xi_{b ; d}+\xi_{d ; b}\right) F_{m}^{h} F_{c}^{m}-g_{b d}\left(F_{m ; \mu}^{h} \xi^{\mu}+F_{\mu}^{h} \xi_{; m}^{\mu}\right. \\
& \left.\left.-F_{m}^{\mu} \xi_{; \mu}^{h}\right) F_{c}^{m}-g_{b d} F_{m}^{h}\left(F_{c ; \mu}^{m} \xi^{\mu}+F_{\mu}^{m} \xi_{; c}^{\mu}-F_{c}^{\mu} \xi_{; \mu}^{m}\right)\right]+\left[\frac { k A } { 4 } \left(F_{i j ; \mu} \xi^{\mu}+F_{i \mu} \xi_{; j}^{\mu}\right.\right. \\
& \left.\left.+F_{j \mu} \xi_{; i}^{\mu}\right) F^{i j}+\frac{k A}{4} F_{i j}\left(F_{; \mu}^{i j} \xi^{\mu}-F^{i \mu} \xi_{; \mu}^{j}-F^{j \mu} \xi_{; j \mu}^{i}\right)-\frac{B}{3} \mathcal{L}_{\xi}(R)\right]\left[\delta_{d}^{h} g_{b c}\right. \\
& \left.-\delta_{c}^{h} g_{b d}\right]+\left(\frac{k A}{4} F_{i j} F^{i j}-\frac{B R}{3}\right)\left[\delta_{d}^{h}\left(\xi_{b ; c}+\xi_{c ; b}\right)-\delta_{c}^{h}\left(\xi_{b ; d}+\xi_{d ; b}\right)\right]
\end{aligned}
$$

using the definition of motion 2.1, Maxwell collineation (2.14), semiconformal curvature collineation, and Lemma 5.1 in equation (5.5). This Completes the proof. 
Further, using equations (5.1) and (5.2) in equation (3.11), we get

$$
\begin{aligned}
P_{b c d}^{h}= & A W_{b c d}^{h}-\frac{k A}{6}\left(\delta_{d}^{h} F_{b m} F_{c}^{m}-\delta_{c}^{h} F_{b m} F_{d}^{m}\right)-\frac{k A}{2}\left(F_{m}^{h} F_{d}^{m} g_{b c}\right. \\
& \left.-F_{m}^{h} F_{c}^{m} g_{b d}\right)+\left(\delta_{d}^{h} g_{b c}-\delta_{c}^{h} g_{b d}\right)\left(\frac{k A}{6} F_{i j} F^{i j}-\frac{B R}{3}\right),
\end{aligned}
$$

which on taking Lie derivative leads to

$$
\begin{aligned}
\mathcal{L}_{\xi} P_{b c d}^{h}= & A \mathcal{L}_{\xi} W_{b c d}^{h}-\frac{k A}{6}\left[\delta_{d}^{h} \mathcal{L}_{\xi}\left(F_{b m}\right) F_{c}^{m}+\delta_{d}^{h} F_{b m} \mathcal{L}_{\xi}\left(F_{c}^{m}\right)-\delta_{c}^{h} \mathcal{L}_{\xi}\left(F_{b m}\right) F_{d}^{m}\right. \\
& \left.-\delta_{c}^{h} F_{b m} \mathcal{L}_{\xi}\left(F_{d}^{m}\right)\right]-\frac{k A}{2}\left[\mathcal{L}_{\xi}\left(F_{m}^{h}\right) F_{d}^{m} g_{b c}+F_{m}^{h} \mathcal{L}_{\xi}\left(F_{d}^{m}\right) g_{b c}+F_{m}^{h} F_{d}^{m} \mathcal{L}_{\xi}\left(g_{b c}\right)\right. \\
& \left.-\mathcal{L}_{\xi}\left(F_{m}^{h}\right) F_{c}^{m} g_{b d}-F_{m}^{h} \mathcal{L}_{\xi}\left(F_{c}^{m}\right) g_{b d}-F_{m}^{h} F_{c}^{m} \mathcal{L}_{\xi}\left(g_{b d}\right)\right]+\left[\delta_{d}^{h} \mathcal{L}_{\xi}\left(g_{b c}\right)\right. \\
& \left.-\delta_{c}^{h} \mathcal{L}_{\xi}\left(g_{b d}\right)\right]\left[\frac{k A}{6} F_{i j} F^{i j}-\frac{B R}{3}\right]+\left[\delta_{d}^{h} g_{b c}-\delta_{c}^{h} g_{b d}\right]\left[\frac{k A}{6} \mathcal{L}_{\xi}\left(F_{i j}\right) F^{i j}\right. \\
& \left.+\frac{k A}{6} F_{i j} \mathcal{L}_{\xi}\left(F^{i j}\right)-\frac{B}{3} \mathcal{L}_{\xi}(R)\right] .
\end{aligned}
$$

Again, using the definitions of Lie derivative for metric and electromagnetic field tensors in equation (5.6), we get

$$
\begin{aligned}
\mathcal{L}_{\xi} P_{b c d}^{h}= & A \mathcal{L}_{\xi} W_{b c d}^{h}-\frac{k A}{6}\left[\delta_{d}^{h}\left(F_{b m ; \mu} \xi^{\mu}+F_{b \mu} \xi_{; m}^{\mu}+F_{m \mu} \xi_{b}^{\mu}\right) F_{c}^{m}+\delta_{c}^{h} F_{b m}\left(F_{c ; \mu}^{m} \xi^{\mu}\right.\right. \\
& \left.+F_{\mu}^{m} \xi_{; c}^{\mu}-F_{c}^{\mu} \xi_{; \mu}^{m}\right)-\delta_{c}^{h}\left(F_{b m ; \mu} \xi^{\mu}+F_{b \mu} \xi_{; m}^{\mu}+F_{m \mu} \xi_{; b}^{\mu}\right) F_{d}^{m}-\delta_{c}^{h} F_{b m}\left(F_{d ; \mu}^{m} \xi^{\mu}\right. \\
& \left.\left.+F_{\mu}^{m} \xi_{; d}^{\mu}-F_{d}^{\mu} \xi_{; \mu}^{m}\right)\right]-\frac{k A}{2}\left[\left(\xi_{b ; c}+\xi_{c ; b}\right) F_{m}^{h} F_{d}^{m}+g_{b c}\left(F_{m ; \mu}^{h} \xi^{\mu}+F_{\mu}^{h} \xi_{; m}^{\mu}\right.\right. \\
& \left.-F_{m}^{\mu} \xi_{; \mu}^{h}\right) F_{d}^{m}+g_{b c} F_{m}^{h}\left(F_{d ; \mu}^{m} \xi^{\mu}+F_{\mu}^{m} \xi_{; d}^{\mu}-F_{d}^{\mu} \xi_{; \mu}^{m}\right)-\left(\xi_{b ; d}+\xi_{d ; b}\right) F_{m}^{h} F_{c}^{m} \\
& \left.-g_{b d}\left(F_{m ; \mu}^{h} \xi^{\mu}+F_{\mu}^{h} \xi_{; m}^{\mu}-F_{m}^{\mu} \xi_{; \mu}^{h}\right) F_{c}^{m}-g_{b d} F_{m}^{h}\left(F_{c ; \mu}^{m} \xi^{\mu}+F_{\mu}^{m} \xi_{; c}^{\mu}-F_{c}^{\mu} \xi_{; \mu}^{m}\right)\right] \\
& +\left[\delta_{d}^{h}\left(\xi_{b ; c}+\xi_{c ; b}\right)-\delta_{c}^{h}\left(\xi_{b ; d}+\xi_{d ; b}\right)\right]\left[\frac{k A}{6} F_{i j} F^{i j}-\frac{B R}{3}\right]+\left[\delta_{d}^{h} g_{b c}-\delta_{c}^{h} g b d\right] \\
& \times\left[\frac{k A}{6}\left(F_{i j ; \mu} \xi^{\mu}+F_{i ; \mu} \xi_{; j}^{\mu}+F_{j ; \mu} \xi_{i ; \mu}^{\mu}\right) F^{i j}+\frac{k A}{6} F_{i j}\left(F_{; \mu}^{i j} \xi^{\mu}-F^{i \mu} \xi_{; \mu}^{j}-F_{\mu}^{j} \xi_{; \mu}^{i}\right)+\frac{B}{3} \mathcal{L}_{\xi}(R)\right] .
\end{aligned}
$$

Moreover, for non-null electromagnetic fields, the semiconformal curvature tensor can be expressed as

$$
\begin{aligned}
P_{b c d}^{h}= & A\left[M_{b c d}^{h}-\frac{k}{2}\left(g_{b c} F_{m}^{h} F_{d}^{m}-\delta_{c}^{h} F_{b m} F_{d}^{m}+\delta_{d}^{h} F_{b m} F_{c}^{m}-g_{b d} F_{m}^{h} F_{c}^{m}\right)\right] \\
& +\left(\frac{k A}{4} F_{i j} F^{i j}+\frac{R A}{12}-\frac{B R}{3}\right)\left(\delta_{d}^{h} g_{b c}-\delta_{c}^{h} g_{b d}\right) .
\end{aligned}
$$

Taking the Lie derivative on both sides of the equation (5.8), we get

$$
\begin{aligned}
\mathcal{L}_{\xi} P_{b c d}^{h}= & A \mathcal{L}_{\xi} M_{b c d}^{h}-\frac{k A}{2}\left[\mathcal{L}_{\xi}\left(g_{b c}\right) F_{m}^{h} F_{d}^{m}+g_{b c} \mathcal{L}_{\xi}\left(F_{m}^{h}\right) F_{d}^{m}+g_{b c} F_{m}^{h} \mathcal{L}_{\xi}\left(F_{d}^{m}\right)\right. \\
& \left.-\delta_{c}^{h} \mathcal{L}_{\xi}\left(F_{b m}\right) F_{d}^{m}-\delta_{c}^{h} F_{b m} \mathcal{L}_{\xi}\left(F_{d}^{m}\right)\right]+\frac{k A}{2}\left[\delta_{d}^{h} \mathcal{L}_{\xi}\left(F_{b m}\right) F_{c}^{m}+\delta_{d}^{h} F_{b m} \mathcal{L}_{\xi}\left(F_{c}^{m}\right)\right. \\
& \left.-\mathcal{L}_{\xi}\left(g_{b d}\right) F_{m}^{h} F_{c}^{m}-g_{b d} \mathcal{L}_{\xi}\left(F_{m}^{h}\right) F_{c}^{m}-g_{b d} F_{m}^{h} \mathcal{L}_{\xi}\left(F_{c}^{m}\right)\right]+\left[\frac { k A } { 4 } \mathcal { L } _ { \xi } \left(F_{i j}\right.\right. \\
& \left.+\frac{k A}{4} F_{i j} \mathcal{L}_{\xi}\left(F^{i j}\right)+\frac{A}{12} \mathcal{L}_{\xi}(R)-\frac{B}{3} \mathcal{L}_{\xi}(R)\right]\left[\delta_{d}^{h} g_{b c}-\delta_{c}^{h} g_{b d}\right] \\
& +\left(\frac{k A}{4} F_{i j} F^{i j}+\frac{R A}{12}-\frac{B R}{3}\right)\left[\delta_{d}^{h} \mathcal{L}_{\xi}\left(g_{b c}\right)-\delta_{c}^{h} \mathcal{L}_{\xi}\left(g_{b d}\right)\right],
\end{aligned}
$$


which on using the definitions of Lie derivative of metric and electromagnetic field tensors leads to

$$
\begin{aligned}
\mathcal{L}_{\xi} P_{b c d}^{h}= & A \mathcal{L}_{\xi} M_{b c d}^{h}-\frac{k A}{2}\left[\left(\xi_{b ; c}+\xi_{c ; b}\right) F_{m}^{h} F_{d}^{m}+g_{b c}\left(F_{m ; \mu}^{h} \xi^{\mu}+F_{\mu}^{h} \xi_{; m}^{\mu}-F_{m}^{\mu} \xi_{; \mu}^{h}\right) F_{d}^{m}\right. \\
& +g_{b c} F_{m}^{h}\left(F_{d ; \mu}^{m} \xi^{\mu}+F_{\mu}^{m} \xi_{; d}^{\mu}-F_{d}^{\mu} \xi_{; \mu}^{m}\right)-\delta_{c}^{h}\left(F_{b m ; \mu} \xi^{\mu}+F_{b \mu} \xi_{; m}^{\mu}+F_{m \mu} \xi_{; b}^{\mu}\right) F_{d}^{m} \\
& \left.-\delta_{c}^{h} F_{b m}\left(F_{d ; \mu}^{m} \xi^{\mu}+F_{\mu}^{m} \xi_{; d}^{\mu}-F_{d}^{\mu} \xi_{; \mu}^{m}\right)\right]-\frac{k A}{2}\left[\delta _ { d } ^ { h } \left(F_{b m ; \mu} \xi^{\mu}+F_{b \mu} \xi_{m}^{\mu}\right.\right. \\
& \left.+F_{m \mu} \xi_{; b}^{\mu}\right) F_{c}^{m}+\delta_{d}^{h} F_{b m}\left(F_{c ; \mu}^{m} \xi^{\mu}+F_{\mu}^{m} \xi_{; c}^{\mu}-F_{c}^{\mu} \xi_{; \mu}^{m}\right)-\left(\xi_{b ; d}+\xi_{d ; b}\right) F_{m}^{h} F_{c}^{m} \\
& \left.-g_{b d}\left(F_{m ; \mu}^{h} \xi^{\mu}+F_{\mu}^{h} \xi_{; m}^{\mu}-F_{m}^{\mu} \xi_{; \mu}^{h}\right) F_{c}^{m}-g_{b d} F_{m}^{h}\left(F_{c ; \mu}^{m} \xi^{\mu}+F_{\mu}^{m} \xi_{; c}^{\mu}-F_{c}^{\mu} \xi_{; \mu}^{m}\right)\right] \\
& +\left[\frac{k A}{4}\left(F_{i j ; \mu} \xi^{\mu}+F_{i \mu} \xi_{; j}^{\mu}+F_{j \mu} \xi_{; i}^{\mu}\right) F^{i j}+\frac{k A}{4} F_{i j}\left(F_{; \mu}^{i j} \xi^{\mu}-F^{i \mu} \xi_{; \mu}^{j}-F^{j \mu} \xi_{; \mu}^{i}\right)\right. \\
& \left.+\frac{A}{12} \mathcal{L}_{\xi}(R)-\frac{B}{3} \mathcal{L}_{\xi}(R)\right]\left[\delta_{d}^{h} g_{b c}-\delta_{c}^{h} g_{b d}\right] \\
& +\left(\frac{k A}{4} F_{i j} F^{i j}+\frac{R A}{12}-\frac{B R}{3}\right)\left[\delta_{d}^{h}\left(\xi_{b ; c}+\xi_{c ; b}\right)-\delta_{c}^{h}\left(\xi_{b ; d}+\xi_{d ; b}\right)\right] .
\end{aligned}
$$

Remark 5.3. Making use of the definition of motion 2.1, Maxwell collineation (2.14), semiconformal curvature collineation (3.2), and Lemma 5.1, equations (5.7) and (5.10) give the similar result as in Theorem 5.2 .

Also it is known that

Lemma 5.4 ([19]). If a non-null electromagnetic field, under certain geometric conditions, admits Maxwell collineation along vector field $\xi$, then $\xi$ is Killing.

Theorem 5.5. A non-null electromagnetic field admits semiconformal curvature collineation if it admits Maxwell collineation.

Proof. From the equations (2.4), (5.7), and (5.10) in the framework of Lemma 5.4 we can establish the proof of the theorem.

Further, the energy momentum tensor $T_{a b}$, for a null electromagnetic field is defined as

$$
\mathrm{T}_{\mathrm{ab}}=\mathrm{F}_{\mathrm{an}} \mathrm{F}_{\mathrm{b}}^{\mathrm{n}} \text {, }
$$

where $F_{i j}=s_{i} t_{j}-t_{i} s_{j}$ and $s_{i} s^{i}=s_{i} t^{i}=0, t_{i} t^{i}=1$, vectors $s$ and $t$ are the propagation and polarization vectors, respectively.

Lemma 5.6 ([2]). A null electromagnetic field admits a Maxwell collineation along the propagation (polarization) vector if the propagation (polarization) vector is Killing and expansion-free.

Theorem 5.7. A null electromagnetic field admits semiconformal curvature collineation along a propagation (polarization) vector if propagation (polarization) vector is killing and expansion-free.

Proof. Making use of the equations (5.2) and (5.11) in equation (3.6), we have

$$
P_{b c d}^{h}=A\left[R_{b c d}^{h}-\frac{k}{2}\left(g_{b c} F_{n}^{h} F_{d}^{n}-\delta_{c}^{h} F_{b n} F_{d}^{n}+\delta_{d}^{h} F_{b n} F_{c}^{n}-g_{b d} F_{n}^{h} F_{c}^{n}\right)\right]-\frac{B R}{3}\left(\delta_{d}^{h} g_{b c}-\delta_{c}^{h} g_{b d}\right) .
$$

Taking Lie derivative of this equation, we get

$$
\mathcal{L}_{\xi} P_{b c d}^{h}=A \mathcal{L}_{\xi} R_{b c d}^{h}-\frac{k A}{2}\left[\mathcal{L}_{\xi}\left(g_{b c}\right) F_{n}^{h} F_{d}^{n}+g_{b c} \mathcal{L}_{\xi}\left(F_{n}^{h}\right) F_{d}^{n}+g_{b c} F_{n}^{h} \mathcal{L}_{\xi}\left(F_{d}^{n}\right)\right.
$$




$$
\begin{aligned}
& \left.-\delta_{c}^{h} \mathcal{L}_{\xi}\left(F_{b n}\right) F_{d}^{n}-\delta_{c}^{h} F_{b n} \mathcal{L}_{\xi}\left(F_{d}^{n}\right)\right]-\frac{k A}{2}\left[\delta_{d}^{h} \mathcal{L}_{\xi}\left(F_{b n}\right) F_{c}^{n}+\delta_{d}^{h} F_{b n} \mathcal{L}_{\xi}\left(F_{c}^{n}\right)\right. \\
& \left.-\mathcal{L}_{\xi}\left(g_{b d}\right) F_{n}^{h} F_{c}^{n}-g_{b d} \mathcal{L}_{\xi}\left(F_{n}^{h}\right) F_{c}^{n}-g_{b d} F_{n}^{h} \mathcal{L}_{\xi}\left(F_{c}^{n}\right)\right]-\frac{B}{3} \mathcal{L}_{\xi}(R)\left[\delta_{d}^{h} g_{b c}-\delta_{c}^{h} g_{b d}\right] \\
& -\frac{B R}{3}\left[\delta_{d}^{h} \mathcal{L}_{\xi}\left(g_{b c}\right)-\delta_{c}^{h} \mathcal{L}_{\xi}\left(g_{b d}\right)\right],
\end{aligned}
$$

which on using the definition of Lie derivative reduces to

$$
\begin{aligned}
\mathcal{L}_{\xi} P_{b c d}^{h}= & A \mathcal{L}_{\xi} R_{b c d}^{h}-\frac{k A}{2}\left[\left(\xi_{b ; c}+\xi_{c ; b}\right) F_{n}^{h} F_{d}^{n}+g_{b c}\left(F_{n ; \mu}^{h} \xi^{\mu}+F_{\mu}^{h} \xi_{; n}^{\mu}-F_{n}^{\mu} \xi_{; \mu}^{h}\right) F_{d}^{n}\right. \\
& +g_{b c} F_{n}^{h}\left(F_{d ; \mu}^{n} \xi^{\mu}+F_{\mu}^{n} \xi_{; d}^{\mu}-F_{d}^{\mu} \xi_{; \mu}^{n}\right)-\delta_{c}^{h}\left(F_{b n ; \mu} \xi^{\mu}+F_{b \mu} \xi_{; n}^{\mu}+F_{n \mu} \xi_{; b}^{\mu}\right) F_{d}^{n} \\
& \left.-\delta_{c}^{h} F_{b n}\left(F_{d ; \mu}^{n} \xi^{\mu}+F_{\mu}^{n} \xi_{; d}^{\mu}-F_{d}^{\mu} \xi_{; \mu}^{n}\right)\right]-\frac{k A}{2}\left[\delta_{d}^{h}\left(F_{b n ; \mu} \xi^{\mu}+F_{b \mu} \xi_{; n}^{\mu}+F_{n \mu} \xi_{; b}^{\mu}\right) F_{c}^{n}\right. \\
& +\delta_{d}^{h} F_{b n}\left(F_{c ; \mu}^{n}+F_{\mu}^{n} \xi_{; c}^{\mu}-F_{c}^{\mu} \xi_{; \mu}^{n}\right)-\left(\xi_{b ; d}+\xi_{d ; b}\right) F_{n}^{h} F_{c}^{n}-g_{b d}\left(F_{n ; \mu}^{h} \xi^{\mu}+F_{\mu}^{h} \xi_{; n}^{\mu}\right. \\
& \left.\left.-F_{n}^{\mu} \xi_{; \mu}^{h}\right) F_{c}^{n}-g_{b d} F_{n}^{h}\left(F_{c ; \mu}^{n} \xi^{\mu}+F_{\mu}^{n} \xi_{; c}^{\mu}-F_{c}^{\mu} \xi_{; \mu}^{n}\right)\right]-\frac{B}{3} \mathcal{L}_{\xi}(R)\left[\delta_{d}^{h} g_{b c}-\delta_{c}^{h} g_{b d}\right] \\
& -\frac{B R}{3}\left[\delta_{d}^{h}\left(\xi_{b ; c}+\xi_{c ; b}\right)-\delta_{c}^{h}\left(\xi_{b ; d}+\xi_{d ; b}\right)\right] .
\end{aligned}
$$

Thus, using the definitions of motion, Maxwell collineation, semiconformal collineation, Lemma 5.6 and equation (5.12), we establish the proof of the Theorem 5.7.

From equations (5.2) and (5.11), equation (3.11) can be expressed as

$$
P_{b c d}^{h}=A\left[W_{b c d}^{h}+\frac{k}{6}\left(\delta_{c}^{h} F_{b n} F_{d}^{n}-\delta_{d}^{h} F_{b n} F_{c}^{n}\right)+\frac{k}{2}\left(F_{n}^{h} F_{c}^{n} g_{b d}-F_{n}^{h} F_{d}^{n} g_{b c}\right)\right]-\frac{B R}{3}\left(\delta_{d}^{h} g_{b c}-\delta_{c}^{h} g_{b d}\right) .
$$

Operating the Lie derivative on this equation, we get

$$
\begin{aligned}
\mathcal{L}_{\xi} P_{b c d}^{h}= & A \mathcal{L}_{\xi} W_{b c d}^{h}+\frac{k A}{6}\left[\delta_{c}^{h} \mathcal{L}_{\xi}\left(F_{b n}\right) F_{d}^{n}+\delta_{c}^{h} F_{b n} \mathcal{L}_{\xi}\left(F_{d}^{n}\right)-\delta_{d}^{h} \mathcal{L}_{\xi}\left(F_{b n}\right) F_{c}^{n}\right. \\
& \left.-\delta_{d}^{h} F_{b n} \mathcal{L}_{\xi}\left(F_{c}^{n}\right)\right]+\frac{k A}{2}\left[\mathcal{L}_{\xi}\left(g_{b d}\right) F_{n}^{h} F_{c}^{n}+g_{b d} \mathcal{L}_{\xi}\left(F_{n}^{h}\right) F_{c}^{n}+g_{b d} F_{n}^{h} \mathcal{L}_{\xi}\left(F_{c}^{n}\right)\right. \\
& \left.-\mathcal{L}_{\xi}\left(g_{b c}\right) F_{n}^{h} F_{d}^{n}-g_{b c} \mathcal{L}_{\xi}\left(F_{n}^{h}\right) F_{d}^{n}-g_{b c} F_{n}^{h} \mathcal{L}_{\xi}\left(F_{d}^{n}\right)\right]-\frac{B}{3} \mathcal{L}_{\xi}(R)\left[\delta_{d}^{h} g_{b c}-\delta_{c}^{h} g_{b d}\right] \\
& -\frac{B R}{3}\left[\delta_{d}^{h} \mathcal{L}_{\xi}\left(g_{b c}\right)-\delta_{c}^{h} \mathcal{L}_{\xi}\left(g_{b d}\right)\right],
\end{aligned}
$$

which on using the definition of Lie derivative of tensors leads to

$$
\begin{aligned}
\mathcal{L}_{\xi} P_{b c d}^{h}= & A \mathcal{L}_{\xi} W_{b c d}^{h}+\frac{k A}{6}\left[\delta_{c}^{h}\left(F_{b n ; \mu} \xi^{\mu}+F_{b \mu} \xi_{;}^{\mu}+F_{n \mu} \xi_{; b}^{\mu}\right) F_{d}^{n}+\delta_{c}^{h} F_{b n}\left(F_{d ; \mu}^{n} \xi^{\mu}\right.\right. \\
& \left.+F_{\mu}^{n} \xi_{; d}^{\mu}-F_{d}^{\mu} \xi_{; \mu}^{n}\right)-\delta_{d}^{h}\left(F_{b n ; \mu} \xi^{\mu}+F_{b \mu} \xi_{; n}^{\mu}+F_{n \mu} \xi_{; b}^{\mu}\right) F_{c}^{n}-\delta_{d}^{h} F_{a n}\left(F_{c ; \mu}^{n} \xi^{\mu}\right. \\
& \left.\left.+F_{\mu}^{n} \xi_{; c}^{\mu}-F_{c}^{\mu} \xi_{; \mu}^{n}\right)\right]+\frac{k A}{2}\left[\left(\xi_{b ; d}+\xi_{d ; b}\right) F_{n}^{h} F_{c}^{n}+g_{b d}\left(F_{n ; \mu}^{h} \xi^{\mu}+F_{\mu}^{h} \xi_{; n}^{\mu}\right.\right. \\
& \left.-F_{n}^{\mu} \xi_{; \mu}^{h}\right) F_{c}^{n}+g_{b d} F_{n}^{h}\left(F_{c ; \mu}^{n}+F_{\mu}^{n} \xi_{; c}^{\mu}-F_{c}^{\mu} \xi_{; \mu}^{n}\right)-\left(\xi_{b ; c}+\xi_{c ; b}\right) F_{n}^{h} F_{d}^{n} \\
& \left.-g_{b c}\left(F_{n ; \mu}^{h} \xi^{\mu}+F_{\mu}^{h} \xi_{; n}^{\mu}-F_{n}^{\mu} \xi_{; \mu}^{h}\right) F_{d}^{n}-g_{b c} F_{n}^{h}\left(F_{d ; \mu}^{n} \xi^{\mu}+F_{\mu}^{n} \xi_{; d}^{\mu}-F_{d}^{\mu} \xi_{; \mu}^{n}\right)\right] \\
& -\frac{B}{3} \mathcal{L}_{\xi}(R)\left[\delta_{d}^{h} g_{b c}-\delta_{c}^{h} g_{b d}\right]-\frac{B R}{3}\left[\delta_{d}^{h}\left(\xi_{b ; c}+\xi_{c ; b}\right)-\delta_{c}^{h}\left(\xi_{b ; d}+\xi_{d ; b}\right)\right] .
\end{aligned}
$$


Further using equations (5.2) and (5.11) in equation (3.14), we get

$$
P_{b c d}^{h}=A M_{b c d}^{h}+\frac{1}{2}\left(\delta_{c}^{h} F_{b n} F_{d}^{n}-\delta_{d}^{h} F_{b n} F_{c}^{n}\right)+\frac{1}{2}\left(g_{b d} F_{n}^{h} F_{c}^{n}-g_{b c} F_{n}^{h} F_{d}^{n}\right)+\left(\frac{A R-4 B R}{12}\right)\left(\delta_{d}^{h} g_{b c}-\delta_{c}^{h} g_{b d}\right) .
$$

Operating Lie derivative on this equation, we have

$$
\begin{aligned}
\mathcal{L}_{\xi} P_{b c d}^{h}= & A \mathcal{L}_{\xi} M_{b c d}^{h}+\frac{1}{2}\left[\delta_{c}^{h} \mathcal{L}_{\xi}\left(F_{b n}\right) F_{d}^{n}+\delta_{c}^{h} F_{b n} \mathcal{L}_{\xi}\left(F_{d}^{n}\right)-\delta_{d}^{h} \mathcal{L}_{\xi}\left(F_{b n}\right) F_{c}^{n}\right. \\
& \left.-\delta_{d}^{h} F_{b n} \mathcal{L}_{\xi}\left(F_{c}^{n}\right)\right]+\frac{1}{2}\left[\mathcal{L}_{\xi}\left(g_{b d}\right) F_{n}^{h} F_{c}^{n}+g_{b d} \mathcal{L}_{\xi}\left(F_{n}^{h}\right) F_{c}^{n}+g_{b d} F_{n}^{h} \mathcal{L}_{\xi}\left(F_{c}^{n}\right)\right. \\
& \left.-\mathcal{L}_{\xi}\left(g_{b c}\right) F_{n}^{h} F_{d}^{n}-g_{b c} \mathcal{L}_{\xi}\left(F_{n}^{h}\right) F_{d}^{n}-g_{b c} F_{n}^{h} \mathcal{L}_{\xi}\left(F_{d}^{n}\right)\right] \\
& -\left(\frac{A-4 B}{12}\right) \mathcal{L}_{\xi}(R)\left[\delta_{d}^{h} g_{b c}-\delta_{c}^{h} g_{b d}\right]-\left(\frac{A R-4 B R}{12}\right)\left[\delta_{d}^{h} \mathcal{L}_{\xi}\left(g_{b c}\right)-\delta_{c}^{h} \mathcal{L}_{\xi}\left(g_{b d}\right)\right],
\end{aligned}
$$

now, using the expressions of $\mathcal{L}_{\xi} \mathrm{g}_{\mathrm{ab}}$ and $\mathcal{L}_{\xi} \mathrm{F}_{\mathrm{ab}}$ above equation can be expressed as

$$
\begin{aligned}
\mathcal{L}_{\xi} P_{b c d}^{h}= & A \mathcal{L}_{\xi} M_{b c d}^{h}+\frac{1}{2}\left[\delta_{c}^{h}\left(F_{b n ; \mu} \xi^{\mu}+F_{b \mu} \xi_{; n}^{\mu}+F_{n \mu} \xi_{;}^{\mu}\right) F_{d}^{n}+\delta_{c}^{h} F_{b n}\left(F_{d ; \mu}^{n} \xi^{\mu}+F_{\mu}^{n} \xi_{; d}^{\mu}\right.\right. \\
& \left.\left.-F_{d}^{\mu} \xi_{; \mu}^{n}\right)-\delta_{d}^{h}\left(F_{b n ; \mu} \xi^{\mu}+F_{b \mu} \xi_{; n}^{\mu}+F_{n \mu} \xi_{; b}^{\mu}\right) F_{c}^{n}-\delta_{d}^{h} F_{b n}\left(F_{c ; \mu}^{n} \xi^{\mu}+F_{\mu}^{n} \xi_{;}^{\mu}-F_{c}^{\mu} \xi_{; \mu}^{n}\right)\right] \\
& +\frac{1}{2}\left[\left(\xi_{b ; d}+\xi_{d ; b}\right) F_{n}^{h} F_{c}^{n}+g_{b d}\left(F_{n ; \mu}^{h} \xi^{\mu}+F_{\mu}^{h} \xi_{; n}^{\mu}-F_{n}^{\mu} \xi_{; \mu}^{h}\right) F_{c}^{n}+g_{b d} F_{n}^{h}\left(F_{c ; \mu}^{n}+F_{\mu}^{n} \xi_{; c}^{\mu}\right.\right. \\
& \left.-F_{c}^{\mu} \xi_{; \mu}^{n}\right)-\left(\xi_{b ; c}+\xi_{c ; b}\right) F_{n}^{h} F_{d}^{n}-g_{b c}\left(F_{n ; \mu}^{h} \xi^{\mu}+F_{\mu}^{h} \xi_{; n}^{\mu}-F_{n}^{\mu} \xi_{; \mu}^{h}\right) F_{d}^{n}-g_{b c} F_{n}^{h}\left(F_{d ; \mu}^{n} \xi^{\mu}\right. \\
& \left.\left.+F_{\mu}^{n} \xi_{; d}^{\mu}-F_{d}^{\mu} \xi_{; \mu}^{n}\right)\right]-\left(\frac{A-4 B}{12}\right) \mathcal{L}_{\xi}(R)\left[\delta_{d}^{h} g_{b c}-\delta_{c}^{h} g_{b d}\right] \\
& -\left(\frac{A R-4 B R}{12}\right)\left[\delta_{d}^{h}\left(\xi_{b ; c} \xi_{c ; b}\right)-\delta_{c}^{h}\left(\xi_{b ; d}+\xi_{d ; b}\right)\right] .
\end{aligned}
$$

Remark 5.8. From the definitions of motion, Maxwell collineation, semiconformal collineation, Lemma 5.6 and equations (5.13) and (5.14), we may get the similar results as in Theorem 5.7.

\section{Acknowledgment}

Authors would like to thank the learned referee(s) and editor for the valuable suggestions and detailed comments that improved our paper.

\section{References}

[1] Abdussattar, B. Dwivedi, On Conharmonic transformations in general relativity, Bull. Calcutta Math. Soc., 88 (1996), 465-470. 1

[2] Z. Ahsan, Collineation in electromagnetic fileds in general relativity-The null field case, Tamkang J. Math., 9 (1978), 237-240. 1, 5, 5.6

[3] Z. Ahsan, Symmetries of the electromagnetic fields in general relativity, Acta Phys. Sinica, 4 (1995), 337-343. 5

[4] Z. Ahsan, A symmetry property of the spacetime of general relativity in terms of space-matter tensor, Braz. J. Phys., 26 (1996), 572-576. 1

[5] Z. Ahsan, N. Ahsan, On a symmetry of the electromagnetic fields, Bull. Calcutta Math. Soc., 94 (2002), 385-388. 1, 5

[6] Z. Ahsan, M. Ali, Symmetries of type D pure radiation fields, Internat. J. Theoret. Phys., 51 (2012), $2044-2055$.

[7] Z. Ahsan, M. Ali, Symmetries of Type N Pure Radiation Fields, Internat. J. Theoret. Phys., 54 (2015), 1397-1407.

[8] Z. Ahsan, M. Ali, Curvature tensor for spacetime of general relativity, Int. J. Geom. Methods Mod. Phys., 14 (2017), 13 pages. 1 
[9] M. Ali, Z. Ahsan, Ricci solitons and symmetries of spacetime manifold of general relativity, Global J. Adv. Res. Classical Modern Geo., 1 (2012), 76-85. 1

[10] C. D. Collinson, Conservation laws in general relativity based upon existence of preferred collineation, Gen. Relativity Gravitation, 1 (1970/71), 137-142. 2

[11] K. L. Duggal, Existence of two Killing vector fieldson the spacetime of general relativity, Tensor (N. S.), 32 (1978), 318-322. 5

[12] L. P. Eisenhart, Riemannian Geometry, Princeton University Press, Princeton, (1926). 2, 2

[13] Y. Ishii, On Conharmonic transformations, Tensor (N. S.), 7 (1957), 73-80. 1

[14] G. H. Katzin, J. Levine, W. R. Davis, Curvature collineation: A fundamental symmetry property of the space-times of general relativity defined by the vanishing Lie derivative of the Riemann curvature tensor, J. Mathematical Phys., 10 (1969), 617-620. 1, 2.4, 2.5, 3

[15] G. H. Katzin, J. Levine, W. R. Davis, Journal of Mathematical Physics, Groups of curvature collineations in Riemannian space-times which admit fields of parallel vectors, 11 (1970), 1578-1580. 1

[16] V. I. Khlebnikov, Gravitational radiation in electromagnetic universes, Classical Quantum Gravity, 3 (1986), 169-173. 5

[17] J. Kim, A type of conformal curvature tensor, Far East J. Math. Sci., 99 (2016), 61-74. 1

[18] J. Kim, On pseudo semiconformally symmetric manifolds, Bull. Korean Math. Soc., 54 (2017), 177-186. 1

[19] H. Michalaski, J. Wainwright, Killing vector fields and the Einstein-Maxwell field equations in general relativity, Gen. Relativity Gravitation, 6 (1975), 289-317. 5, 5.1, 5.4

[20] E. Newman, R. Penrose, An approach to gravitational radiation by a method of spin coefficients, J. Mathematical Phys., 3 (1962), 566-578. 5

[21] J. A. Schouten, Ricci Calculus: An Introduction to Tensor Analysis and its geometrical Applications, Springer-Verlag, Berlin-Göttingen-Heidelberg, (1954). 2

[22] A. A. Shaikh, S. K. Hui, On Weakly conharmonically symmetric manifolds, Tensor (N. S.), 70 (2008), 119-134. 1

[23] S. A. Siddiqui, Z. Ahsan, Conharmonic curvature tensor and the spacetime of General Relativity, Differ. Geom. Dyn. Syst., 12 (2010), 213-220. 1, 3.2

[24] N. Tariq, B. O. J. Tupper, A class of algebraically general solutions of the Einstein-Maxwell equations for non-null electromagnetic field, Gen. Relativity Gravitation, 6 (1975), 345-360. 5

[25] T. Y. Thomas, The Differential Invariants of Generalized Spaces, Cambridge University Press, Cambridge, (1934). 2.4

[26] M. L. Woolley, The structure of groups of motions admitted by Einstein-Maxwell space-times, Comm. Math. Phys., 31 (1973), 75-81. 5

[27] K. Yano, Concircular Geometry I. Concircular Transformation, Proc. Imp. Acad. Tokyo, 16 (1940), 195-200. 3

[28] K. Yano, The Theory of Lie Derivatives and its Applications, North-Holland Publishing Co., Amsterdam, (1957). 2, $2.5,3.3$ 